\title{
Optimal Investment and Consumption for an Insurer with High-Watermark Performance Fee
}

\author{
Lin $\mathrm{Xu},{ }^{1}$ Hao Wang, ${ }^{1}$ and Dingjun Yao ${ }^{2}$ \\ ${ }^{1}$ School of Mathematics and Computer Science, Anhui Normal University, Wuhu, Anhui 241000, China \\ ${ }^{2}$ School of Finance, Nanjing University of Finance and Economics, Nanjing, Jiangsu 210023, China \\ Correspondence should be addressed to Lin Xu; xulinahnu@gmail.com
}

Received 18 August 2015; Accepted 5 October 2015

Academic Editor: Xinguang Zhang

Copyright (c) 2015 Lin Xu et al. This is an open access article distributed under the Creative Commons Attribution License, which permits unrestricted use, distribution, and reproduction in any medium, provided the original work is properly cited.

\begin{abstract}
The optimal investment and consumption problem is investigated for an insurance company, which is subject to the payment of high-watermark fee from profit. The objective of insurance company is to maximize the expected cumulated discount utility up to ruin time. The consumption behavior considered in this paper can be viewed as dividend payment of the insurance company. It turns out that the value function of the proposed problem is the viscosity solution to the associated HJB equation. The regularity of the viscosity is discussed and some asymptotic results are provided. With the help of the smooth properties of viscosity solutions, we complete the verification theorem of the optimal control policies and the potential applications of the main result are discussed.
\end{abstract}

\section{Introduction}

Investment and consumption are of great importance in the study of finance and financial engineering. This is due to the fact that investment and consumption not only are the key topic of financial agents but also provide idea and method of deriving equilibrium price of financial derivatives (cf. Shreve and Soner [1]). Applications of stochastic optimal control to management and financial problems were developed from the 1970 s, especially after the papers by Merton [2,3] on portfolio selection. The model and results of Merton were then extended by many authors; for example, see Zariphopoulou [4], Øksendal and Sulem [5], and Fleming and Pang [6]. These problems are also studied in the monograph by Karatzas and Shreve [7]. The decision makers associated with optimal investment and consumption problems that appeared in aforementioned papers stand on the perspective of financial firms or investment bank, and the business income of decision makers comes from proper construction of portfolio positions. Usually, it assumes that the financial market is frictionless: no transaction cost and no tax payment, money that can be infinitely divided, no restrictions on short or long positions, and so forth. More recently, there is also a large, more recent literature related to the investment in markets with frictions. A transaction cost is a typical example. For example, Davis and Norman [8] studied portfolio selection problem with transaction cost, which uses the variance of the portfolio positrons as the risk measure; Janeček and Sîrbu [9] studied the future trading problem with transaction cost. Shreve and Soner [1] investigated optimal consumption and investment with transaction cost; the optimization goal therein is to maximize expected cumulated discounted utility in an infinite time horizon. Whalley and Wilmott [10] studied optimal hedging model with transaction costs. Previously mentioned papers are just a few examples of the growing literature on the topic; for more progress on this topic, readers are also referred to the works of Cvitanić and Karatzas [11], Liu and Loewenstein [12], Korn [13], and Obizhaeva and Wang [14].

Among all kinds of transaction cost, the high gain tax payment or high-watermark fee has attracted many attentions recently. The high-watermark fee is taken as the following rule: whenever the maximum up to today, the so-called high-watermark, exceeds the previously attained historic maximum, the fixed proportion of the profit (relative to the previous maximum) is charged by the fund manager. In the early 1980s, Stiglitz [15] discussed the possibility and necessity of charging high gain tax from investment income. The past two decades have witnessed an increasing attention to the 
research of optimal control problem with high-watermark fee. For example, Dammon et al. [16] investigated optimal investment and consumption problem with capital gain taxes, Goetzmann et al. [17] studied the hedge fund management when charging high gain tax, and Guasoni and Wang [18] studied high-watermark and separation of private problems. As it was shown previously, investment and consumption problem is an important topic for insurer and also a key topic of insurance mathematics or financial mathematics. Thus, it is natural to consider optimal investment and consumption problem for an insurer when high-watermark fees are charged. Up to now, seldom insurance company considers consumption problems. Thus, to discuss optimal consumption for an insurer seems weird. However, an analogue problem in insurance company is the dividend payment or pension payment problem. For example, Højgaard and Taksar [19] studied reinsurance and dividend with transaction costs, Cairns [20] studied optimal pension fund schedule problem, Zhu [21] took both investment and dividend into account in searching for optimal policies, and He and Liang [22] investigated pension schedule and asset allocation problem. For other works on investment and consumption problem related to insurance affair or partially related to insurance affairs, see Bielecki and Pliska [23], Dai and Yi [24], and Young [25].

In the paper of Janeček and Sîrbu [9], the optimal investment and consumption problems for a fund manager on infinite time horizon are considered when the fund manager is subject to the high-watermark fee from investment. However, the model considered there is not suitable for an insurance company because the latter one has premium income and claims with addition to the investment profit (or underlying risk). This paper contributes to bridging this gap. The surplus process of the insurer is specified by a classical risk model and the insurer has the chance to invest into risky asset and risk-free bond market. Whenever the profit of the insurer attains a new maximum, the highwatermark fee is taken as a kind of gain tax. The goal of the insurer is to choose optimal investment and consumption policies before ruin occurs. We will point out that although it seems weird to allow the insurance company to make consumption policies, dividend payment is a very common decision policy for the managers of insurance company. Thus, the consumption framework considered in this paper can be regarded as a kind of dividend payment of insurer. The progress achieved in this paper can be summarized as follows. The optimization problem considered in this paper is relevant to a jump diffusion process. Thus, the associated HJB equation contains an integration part, which brings us some difficulties in proving the smooth properties of the solution to HJB equation. Similarly, it brings us difficulties in proving that the value function is the viscosity solution to $\mathrm{HJB}$ equation with integration part. Following the idea presented in Janeček and Sîrbu [9], we prove that the value function is smooth on its domain. We obtain the value function of an insurer without high-watermark fee, which is not considered in other literature. We obtain a verification theorem, which means that the viscosity solution to HJB equation is indeed the value function. Due to the natural connections between the viscosity solution to $\mathrm{HJB}$ equation and the numerical algorithm to the stochastic control problem, the main result obtained in this paper is useful in the design of the numerical approximating method of the related HJB equation.

The rest of this paper is organized as follows. In Section 2, the model and problem are presented and efforts are made to transform the model such that dynamic programming principle and HJB equation method are applicable. In Section 3, the definition of viscosity solution to a kind of second-order partial integrodifferential equation is given, and the value function is proved to be the viscosity solution of the associated $\mathrm{HJB}$ equation. In Section 4, by employing the same method of Janeček and Sirbu [9], the viscosity solution is proved to be smooth on certain domain. The properties of viscosity on some singular point are also discussed. Section 5 presents a verification theorem, which asserts that the solution to $\mathrm{HJB}$ equation is indeed the value function and the corresponding feedback control replicates the optimal realization of the insurer. Section 6 concludes the main contributions of this paper and potential applications of our results.

\section{Model and Problem}

2.1. Classical Risk Model and Its Diffusion Approximation. In this subsection, we briefly introduce the classical risk model of an insurance company and its diffusion approximation. The content presented here will be helpful for our later investigation. Classical risk model for an insurer is (cf. Grandell [26])

$$
d U_{t}=a d t-d\left(\sum_{i=1}^{N_{t}} Y_{i}\right)
$$

where $a$ is the constant premium income rate and $\left\{N_{t} ; t \geq 0\right\}$ denotes the number of claims that arrived up to time $t$, which is assumed to be a homogeneous Poisson process with intensity $\lambda_{0}>0$. The individual claims $Y_{1}, Y_{2}, \ldots$ are assumed to be a sequence of independent and identically distributed (i.i.d.) positive random variables (r.v.s.) with common distribution function $G(y)$ and finite expectation and satisfy $G(0)=0$. In addition, it assumes that $\left\{N_{t} ; t \geq 0\right\}$ and $\left\{Y_{i} ; i=1,2, \ldots\right\}$ are mutually independent. For notation convenience, we denote by $\left\{S_{t} ; t \geq 0\right\}$ the aggregate claim process; that is, $S_{t}=\sum_{i=1}^{N_{t}} Y_{i}$. We denote that $\mathbb{E} Y_{i}=\mu$ and $\operatorname{Var}\left(Y_{i}\right)=\sigma^{2}$. Main topic associated with classical risk model is the ruin probability; in mathematics, it is $\mathbb{P}(\tau<\infty)$, where $\tau=\inf \left\{t \geq 0, U_{t}<\right.$ $0\}$ is known as "ruin time" in risk theory. There are many methods to study the ruin time and ruin probability, such as renewal method prompted by Feller and martingale approach introduced by Gerber (cf. Grandell [26]). Another idea is to approximate the classical risk model by some stochastic process with good statistical properties, such as Gaussian process. This is so-called diffusion approximation of classical risk model; see Chapter 1 of Grandell [26].

\subsection{A General Model of Profits from Dynamic Investment in} a Hedge Fund. Now, suppose that the insurance company invests in a risky fund with a share or unit price $F_{t}$ at time $t$. If the insurance company chooses to hold $\theta_{t}$ capital in the 
fund at time $t$ and no fees of any kind are imposed, then the accumulated profit at time $t$, denoted by $P_{t}$, evolves as

$$
\begin{aligned}
d P_{t} & =\theta_{t} \frac{d F_{t}}{F_{t}}+a d t-d\left(\sum_{i=1}^{N(t)} Y_{i}\right)=\theta_{t} \frac{d F_{t}}{F_{t}}+a d t-d S_{t}, \quad 0 \leq t<\infty \\
P_{0} & =0
\end{aligned}
$$

Remark 1. The assumption $P_{0}=0$ seems unreasonable from practice; however, we want to compare our model with the model studied in Janeček and Sîrbu [9], so we made such an assumption. In later discussion, the initial surplus of the insurer is assumed to be $x>0$. is,

Denote by $\left\{M_{t}, t \geq 0\right\}$ the maximum profit process; that

$$
M_{t} \triangleq \sup _{0 \leq s \leq t} P_{s}
$$

Assume now that the manager tracks the high-watermark fee once the insurance company achieves new maximum of profit; the rule is as follows: anytime the high-watermark increases, $\lambda>0$ percentage of this increase is paid to the fund manager. More precisely, the insurance company pays $\lambda \Delta M_{t}=\lambda\left(M_{t+\Delta t}-M_{t}\right)$ to the manager in the interval $[t, t+\Delta t]$. Under such a high-watermark fee taking rule, the evolution equation for the profit $P_{t}$ is revised as

$$
\begin{aligned}
d P_{t} & =\theta_{t-} \frac{d F_{t}}{F_{t}}+a d t-d S_{t}-\lambda d M_{t}, \quad P_{0}=0, \\
M_{t} & =\sup _{0 \leq s \leq t} P_{s} .
\end{aligned}
$$

Suppose that the insurance company has an initial maximum profit $i(i \geq 0)$; the profits of the insurance company will be taxed when $P$ reaches value $i$ and will not be taxed before $P$ reaches at least value $i$. Then, for any given $i \geq 0$, the dynamic of $P$ is given by

$$
\begin{aligned}
& d P_{t}=\theta_{t-} \frac{d F_{t}}{F_{t}}+a d t-d S_{t}-\lambda d M_{t}, \quad P_{0}=0, \\
& M_{t}=\sup _{0 \leq s \leq t}\left(P_{s} \vee i\right) .
\end{aligned}
$$

A similar representation appears in the appendix of Guasoni and Wang [18], where an optimization problem related to maximizing utility of the fund manager is studied, which is opposed to the utility of the investor in our case. However, their state equation is similar to (5), so we resort to the same pathwise representation.

Proposition 2. Assume that the share/unit prices process $F$ is a continuous and strictly positive semimartingale, and the predictable processes $\left\{\theta_{t} ; t \geq 0\right\}$ are such that the accumulated profit process corresponding to the trading strategy $\theta$, in case no profit fees are imposed, namely,

$$
I_{t}=a t-S_{t}+\int_{0}^{t} \theta_{u-} \frac{d F_{u}}{F_{u}}, \quad 0 \leq t<\infty,
$$

is well defined. Then (5) has a unique solution, which can be represented pathwise by

$$
\begin{gathered}
P_{t}=I_{t}-\frac{\lambda}{1+\lambda} \sup _{0 \leq s \leq t}\left[I_{s}-i\right]^{+}, \quad 0 \leq t<\infty, \\
M_{t}=i+\frac{1}{1+\lambda} \max _{0 \leq s \leq t}\left[I_{s}-i\right]^{+}, \quad 0 \leq t<\infty .
\end{gathered}
$$

Proof. Note that $P_{0}=I_{0}=0, M_{0}=i$; (5) can be rewritten as

$$
\left(P_{t}-i\right)+\lambda \sup _{0 \leq s \leq t}\left[P_{s}-i\right]^{+}=\left(I_{t}-i\right), \quad 0 \leq t<\infty .
$$

Taking the positive part and the supremum on both sides, it follows that

$$
\begin{aligned}
(1+\lambda)\left(M_{t}-i\right) & =(1+\lambda) \sup _{0 \leq s \leq t}\left[P_{s}-i\right]^{+} \\
& =\sup _{0 \leq s \leq t}\left[I_{s}-i\right]^{+}, \quad 0 \leq t<\infty .
\end{aligned}
$$

Replacing (10) into (5), we finish the proof of uniqueness.

By checking that the process in (7) is a solution of (5), more precisely,

$$
\begin{aligned}
d P_{t} & =d\left(I_{t}-\frac{\lambda}{1+\lambda} \sup _{0 \leq s \leq t}\left[I_{s}-i\right]^{+}\right) \\
& =d I_{t}-\frac{\lambda}{1+\lambda} d\left(\sup _{0 \leq s \leq t}\left[I_{s}-i\right]^{+}\right) \\
& =\theta_{t-} \frac{d F_{t}}{F_{t}}+a t-S_{t}-\lambda d M_{t} .
\end{aligned}
$$

This completes the proof.

2.3. Optimal Investment and Consumption in a Special Model. Assume that the insurance company starts with initial capital $x>0$ and only additional investment opportunity is the money market paying zero interest rate. The insurance company is given the intimal high-watermark $i \geq 0$ for its profits. We assume that the insurance company consumes at a rate $\gamma_{t}>0$ per unit of time. Consumption can be made either from the money market account or from accumulated profit. Denote by

$$
C_{t} \triangleq \int_{0}^{t} \gamma_{s} d s, \quad 0 \leq t<\infty,
$$

the accumulated consumption process and by $X_{t}^{\theta, \gamma}$ the wealth process of an insurer associated with decision policy $(\theta, \gamma)$. Since the money market pays zero interest rate, the wealth $X_{t}^{\theta, \gamma}$ is formulated as

$$
X_{t}^{\theta, \gamma}=x+P_{t}-C_{t}, \quad 0 \leq t \leq \tau^{\theta, \gamma},
$$

where $\tau^{\theta, \gamma} \triangleq \inf \left\{s \geq 0: X_{s}^{\theta, \gamma} \leq 0\right\}$ is the first time that the wealth goes below zero. In actuarial theory, $\tau$ is referred to as the "ruin time." In later discussion, for notation ease, we drop the symbol $\theta, \gamma$ in $\tau^{\theta, \gamma}$. 
If consumption is taken into account, the high-watermark of the insurance company's profit can be represented as

$$
\begin{aligned}
M_{t}^{\theta, \gamma} & =\sup _{0 \leq s \leq t}\left[\left(X_{s}^{\theta, \gamma}+C_{s}-x\right) \vee i\right]^{+} \\
& =i+\sup _{0 \leq s \leq t}\left[\left(X_{s}^{\theta, \gamma}+C_{s}\right)-k\right]^{+},
\end{aligned}
$$

where $k \triangleq x+i \geq x>0$. In this situation, wealth evolves as

$$
\begin{aligned}
& d X_{t}^{\theta, \gamma}=\theta_{t-} \frac{d F_{t}}{F_{t}}+a d t-\gamma_{t} d t-d S_{t}-\lambda d M_{t}^{\theta, \gamma}, \\
& X_{0}^{\theta, \gamma}=x, \\
& M_{t}^{\theta, \gamma}=i+\sup _{0 \leq s \leq t}\left[\left(X_{s}^{\theta, \gamma}+\int_{0}^{s} \gamma_{u} d u\right)-k\right]^{+}, \\
& 0 \leq t \leq \tau .
\end{aligned}
$$

So far, this is a general model of investment/consumption in a hedge fund, which is also a good model of taxation. In what follows, we focus on a simple but important case, where the fund share/unit price $\left\{F_{t}, t \geq 0\right\}$ evolves as a geometric Brownian motion; that is,

$$
\frac{d F_{t}}{F_{t}}=\alpha d t+\sigma d W_{t}, \quad 0 \leq t<\infty
$$

where $\left(W_{t}\right)_{0 \leq t<\infty}$ is a standard Brownian motion defined on the filtered probability space $\left(\Omega, \mathscr{F},\left(\mathscr{F}_{t}\right)_{0 \leq t<\infty}, \mathbb{P}\right)$. With this notation, (15) becomes

$$
\begin{array}{r}
d X_{t}^{\theta, \gamma}=\left(\theta_{t} \alpha-\gamma_{t}+a\right) d t+\theta_{t} \sigma d W_{t}-d S_{t}-\lambda d M_{t}^{\theta, \gamma}, \\
X_{0}^{\theta, \gamma}=x, \\
M_{t}^{\theta, \gamma}=i+\sup _{0 \leq s \leq t}\left[\left(X_{s}^{\theta, \gamma}+\int_{0}^{s} \gamma_{u} d u\right)-k\right]^{+}, \\
0 \leq t \leq \tau .
\end{array}
$$

In order to use dynamic programming, we want to represent the control problem using a state process of minimal dimension. What is more, since we want to apply the HJB equation method, it is necessary to embed our state process into a Markovian system. As usual, the wealth $X$ has to be a part of the state. But using $(X, M)$ as state is not a possibility, since $M$ does not contain the information on past consumption, just that on past profits. Copying the method of Janeček and Sîrbu [9], we observed that the fee is being paid as soon as the current profit $P_{t}^{\theta, \gamma}=X_{t}^{\theta, \gamma}+C_{t}-x$ (current wealth plus accumulated consumption plus aggregate claim minus income minus initial wealth) hits the high-watermark $M_{t}^{\theta, \gamma}=i+\sup _{0 \leq s \leq t}\left[X_{s}+C_{s}-k\right]^{+}$. In other words, fees are paid whenever

$$
X_{t}^{\theta, \gamma}+C_{t}-k=\sup _{0 \leq s \leq t}\left[\left(X_{s}+C_{s}\right)-k\right]^{+}
$$

which is the same as $X^{\theta, \gamma}=K^{\theta, \gamma}$ for

$$
\begin{aligned}
K_{t}^{\theta, \gamma} & \triangleq k+\sup _{0 \leq s \leq t}\left[\left(X_{s}^{\theta, \gamma}+C_{s}\right)-k\right]^{+}-C_{t} \\
& =\sup _{0 \leq s \leq t}\left[\left\{X_{s}^{\theta, \gamma}+C_{s}\right\} \vee k\right]-C_{t} \geq X_{t}^{\theta, \gamma} .
\end{aligned}
$$

We now choose as state process the two-dimensional process $(X, K)$ which satisfies $X \leq K$ and is reflected whenever $X=$ $K$. The controlled state process $(X, K)$ follows the evolution

$$
\begin{aligned}
d X_{t}^{\theta, \gamma}= & \left(\theta_{t} \alpha-\gamma_{t}+a\right) d t+\theta_{t} \sigma d W_{t}-d S_{t} \\
& -\lambda\left(d K_{t}^{\theta, \gamma}+\gamma_{t} d t\right), \quad X_{0}=x, \\
K_{t}^{\theta, \gamma}= & \sup _{0 \leq s \leq t}\left[\left\{X_{s}^{\theta, \gamma}+\int_{0}^{s} \gamma_{u} d u\right\} \vee k\right]-\int_{0}^{t} \gamma_{u} d u, \\
& 0 \leq t \leq \tau .
\end{aligned}
$$

Equation (20) is implicit, as is (5). The pathwise representation in Proposition 2 can be easily translated into a pathwise solution (20). More precisely, we have Proposition 3, and the proof of Proposition 3 is similar to Proposition 2; we omit it here.

Proposition 3. Assume that the predictable process $(\theta, \gamma)$ satisfies

$$
\mathbb{P}\left(\int_{0}^{t}\left(\left|\theta_{u}\right|^{2}+\gamma_{u}\right) d u<\infty, \forall 0 \leq t \leq \tau\right)=1
$$

Denote

$$
\begin{aligned}
& Z_{t}=\int_{0}^{t} \theta_{u}\left(\alpha d u+\sigma d W_{u}\right)+a t-S_{t}, \\
& C_{t}=\int_{0}^{t} \gamma_{u} d u, \quad 0 \leq t \leq \tau .
\end{aligned}
$$

Suppose that the accumulated profit process corresponding to the trading strategy $\theta$ is well defined. Then

$$
\begin{aligned}
& X_{t}^{\theta, \gamma}=x+Z_{t}-C_{t}-\frac{\lambda}{1+\lambda} \sup _{0 \leq s \leq t}\left[Z_{s}-i\right]^{+}, \\
& \quad 0 \leq t \leq \tau, 0 \leq t \leq \tau . \\
& K_{t}^{\theta, \gamma}=k+\frac{1}{1+\lambda} \sup _{0 \leq s \leq t}\left[Z_{s}-i\right]^{+}-C_{t}, \quad 0 \leq t
\end{aligned}
$$

The high-watermark is computed as

$$
M_{t}^{\theta, \gamma}=i+\frac{1}{1+\lambda} \sup _{0 \leq s \leq t}\left[Z_{s}-i\right]^{+}, \quad 0 \leq t \leq \tau .
$$

Fix an initial capital $x>0$ and an initial high-watermark of profits $i \geq 0$. Recall that $k \triangleq x+i \geq x$. An investment/ consumption strategy $(\theta, \gamma)$ is called admissible with respect to the initial date $(x, k)$ if it satisfies integrability conditions (21); the consumption stream is positive $\left(\gamma_{t} \geq 0\right)$. We denote by $\mathscr{A}(x, k)$ the set of all admissible strategies at $(x, k)$. 
We consider a concave utility function $U:(0, \infty) \rightarrow$ $\mathbb{R}$. So we can define the expected utility from consumption $\mathbb{E}\left[\int_{0}^{\tau} e^{-\beta t} U\left(\gamma_{t}\right) d t\right]$. The object of this paper is to research the optimal investment/consumption amounts $(\theta, \gamma)$ related to following optimization problem (for each fixed $(x, k)$ ):

$$
v(x, k) \triangleq \sup _{(\theta, \gamma) \in \mathscr{A}(x, k)} \mathbb{E}\left[\int_{0}^{\tau} e^{-\beta t} U\left(\gamma_{t}\right) d t\right],
$$

$$
0 \leq x \leq k
$$

One should note that once the initial surplus is negative, that is, $x<0$, it immediately followed by

$$
v(x, k)=0, \quad \forall x<0 .
$$

Function $v$ defined above is called the value function. We further assume that the utility function $U$ has the particular form

$$
U(\gamma)=\frac{\gamma^{1-p}}{1-p}, \quad \gamma>0,
$$

for some $p>0, p \neq 1$, where $p$ is called the relative risk aversion coefficient.

Using the controls $(\theta, \gamma)$ the insurance company controls the process $(X, K)$ in $(21)$ which is restricted to the domain $0<x \leq k$ and is reflected on the diagonal $x=k$ in the direction given by the vector

$$
\widetilde{r} \triangleq\left(\begin{array}{c}
-\lambda \\
1
\end{array}\right)
$$

So, state (20) can be rewritten as

$$
\begin{aligned}
d\left(\begin{array}{c}
X_{t}^{\theta, \gamma} \\
K_{t}^{\theta, \gamma}
\end{array}\right)= & \left(\begin{array}{c}
\left(\theta_{t} \alpha-\gamma_{t}+a\right) d t+\theta_{t} \sigma d W_{t}-d S_{t} \\
-\gamma_{t} d t
\end{array}\right) \\
& +\tilde{\gamma} d M_{t}^{\theta, \gamma}, \quad 0 \leq t \leq \tau,
\end{aligned}
$$

where

$$
\int_{0}^{t} 1_{\left\{X_{s}^{\theta, \gamma} \neq K_{s}^{\theta, \gamma}\right\}} d M_{s}=0
$$

Denote the continuous part of process $\left(X_{t}^{\theta, \gamma}, K_{t}^{\theta, \gamma}\right)$ by $\left(X_{t}^{c, \theta, \gamma}, K_{t}^{c, \theta, \gamma}\right)$; that is,

$$
\begin{aligned}
d\left(\begin{array}{c}
X_{t}^{c, \theta, \gamma} \\
K_{t}^{c, \theta, \gamma}
\end{array}\right)= & \left(\begin{array}{c}
\left(\theta_{t} \alpha-\gamma_{t}+a\right) d t+\theta_{t} \sigma d W_{t} \\
-\gamma_{t} d t
\end{array}\right) \\
& +\tilde{\gamma} d M_{t}, \theta, \gamma, \quad 0 \leq t \leq \tau .
\end{aligned}
$$

The main goal of the present paper is to analyze the impact of fees on the investment/consumption strategies and the main method in this paper relies on HJB equation. One should note that, with the introduction of process $K_{t}^{\theta, \gamma}$, we embed our model into a Markov system, which enables further discussion.

\section{Dynamic Programming and HJB Equation}

Now, in order to obtain the HJB equation as follows, we will use the dynamic programming principle; see Proposition 4. The proof of Proposition 4 is similar to the one in Azcue and Muler [27] and we omit the proof here.

Proposition 4. Suppose that $(\widehat{\theta}, \widehat{\gamma})$ is an optimal control. Then one has

$$
\begin{aligned}
& v(x, k) \\
& =\mathbb{E}\left[\int_{0}^{h \wedge \tau} e^{-\beta s} U\left(\widehat{\gamma}_{s}\right) d s+e^{-\beta(h \wedge \tau)} v\left(X_{h \wedge \tau}^{\hat{\theta}, \widehat{\gamma}}, K_{h \wedge \tau}^{\hat{\theta}, \widehat{\gamma}}\right)\right], \\
& v\left(X_{\tau}^{\hat{\theta}, \hat{\gamma}}, K_{\tau}^{\hat{\theta}, \hat{\gamma}}\right)=0 .
\end{aligned}
$$

If $v(x, n)$ is smooth enough, by Itô's Lemma, we have

$$
\begin{aligned}
e^{-\beta(h \wedge \tau)} v\left(X_{h \wedge \tau}^{\hat{\theta}, \hat{\gamma}}, K_{h \wedge \tau}^{\hat{\theta}, \hat{\gamma}}\right)=v(x, k) \\
+\int_{0}^{h \wedge \tau}\left(-\beta e^{-\beta s} v\right) d s+\int_{0}^{h \wedge \tau} e^{-\beta s} \frac{\partial v}{\partial x} d X_{s}^{c} \\
+\int_{0}^{h \wedge \tau} e^{-\beta s} \frac{\partial v}{\partial k} d K_{s}^{c}+\frac{1}{2} \int_{0}^{h \wedge \tau} e^{-\beta s} \frac{\partial^{2} v}{\partial x^{2}} d X_{s}^{c} d X_{s}^{c}+\frac{1}{2} \\
\cdot \int_{0}^{h \wedge \tau} e^{-\beta s} \frac{\partial^{2} v}{\partial k^{2}} d K_{s}^{c} d K_{s}^{c}+\frac{1}{2} \\
\cdot \int_{0}^{h \wedge \tau} e^{-\beta s} \frac{\partial^{2} v}{\partial x \partial k} d X_{s}^{c} d K_{s}^{c}+\int_{0}^{h \wedge \tau} \int_{0}^{\infty} e^{-\beta s} \\
\cdot\left[v\left(X_{s-}^{\widehat{\theta}, \widehat{\gamma}}-Y, K_{s-}^{\hat{\theta}, \widehat{\gamma}}\right)-v\left(X_{s-}^{\widehat{\theta}, \hat{\gamma}}, K_{s-}^{\hat{\theta}, \hat{\gamma}}\right)\right] \bar{K}(d Y, d s)
\end{aligned}
$$

where $\bar{N}$ is the Poisson random measure on $[0, \tau] \times[0, \infty)$ defined by

$$
\bar{K}=\sum_{n \geq 1} \delta\left(T_{k}, Y_{k}\right)
$$

Denote by $\mathscr{L}^{\theta, \gamma} v(\cdot, \cdot)$ (associated with $(\theta, \gamma)$ ) the second-order partial differential operator with the form of

$$
\mathscr{L}^{\theta, \gamma} v=\left(\theta_{t} \alpha-\gamma_{t}+a\right) v_{x}+\frac{1}{2} \theta_{t}^{2} \sigma^{2} v_{x x}-\gamma_{t} v_{k}
$$

where $v_{x}, v_{x x}, v_{k}$ are the first, the second, and the first partial derivatives with respect to $x$ and $k$, respectively. Then, by compensating (33) with

$$
\begin{gathered}
\lambda_{0} \int_{0}^{h \wedge \tau} \int_{0}^{\infty} e^{-\beta s}\left[v\left(X_{s-}^{\widehat{\theta}, \widehat{\gamma}}-Y, K_{s-}^{\widehat{\theta}, \widehat{\gamma}}\right)\right. \\
\left.-v\left(X_{s^{-}}^{\hat{\theta}, \widehat{\gamma}}, K_{s^{-}}^{\widehat{\theta}, \widehat{\gamma}}\right)\right] d G(Y) d s,
\end{gathered}
$$


(33) can be rewritten as

$$
\begin{aligned}
& e^{-\beta(h \wedge \tau)} v\left(X_{h \wedge \tau}^{\widehat{\theta}, \widehat{\gamma}}, K_{h \wedge \tau}^{\widehat{\theta}, \widehat{\gamma}}\right)=v(x, k)+\int_{0}^{h \wedge \tau} e^{-\beta s}(-\beta v \\
& \left.+\mathscr{L}^{\hat{\theta}, \widehat{\gamma}} v\right)\left(X_{s}^{\hat{\theta}, \hat{\gamma}}, K_{s}^{\hat{\theta}, \widehat{\gamma}}\right) d s \\
& +\int_{0}^{h \wedge \tau} e^{-\beta s} \widehat{\theta}_{s} \sigma v_{x}\left(X_{s}^{\hat{\theta}, \widehat{\gamma}}, K_{s}^{\widehat{\theta}, \widehat{\gamma}}\right) d W_{s} \\
& +\int_{0}^{h \wedge \tau} e^{-\beta s}\left(-\lambda v_{x}+v_{k}\right)\left(X_{s}^{\hat{\theta}, \hat{\gamma}}, K_{s}^{\hat{\theta}, \widehat{\gamma}}\right) d M_{s} \\
& +\int_{0}^{h \wedge \tau} \int_{0}^{\infty} e^{-\beta s}\left[v\left(X_{s-}^{\widehat{\theta}, \widehat{\gamma}}-Y, K_{s^{-}}^{\widehat{\theta}, \widehat{\gamma}}\right)\right. \\
& \left.-v\left(X_{s^{-}}^{\widehat{\theta}, \widehat{\gamma}}, K_{s^{-}}^{\theta, \gamma}\right)\right]\left[\bar{K}(d Y d s)-\lambda_{0} d G(Y) d s\right]
\end{aligned}
$$

$$
\begin{aligned}
& +\lambda_{0} \int_{0}^{h \wedge \tau} \int_{0}^{\infty} e^{-\beta s}\left[v\left(X_{s-}^{\widehat{\theta}, \widehat{\gamma}}-Y, K_{s-}^{\widehat{\theta}, \widehat{\gamma}}\right)\right. \\
& \left.-v\left(X_{s-}^{\hat{\theta}, \widehat{\gamma}}, K_{s^{-}}^{\hat{\theta}, \widehat{\gamma}}\right)\right] d G(Y) d s .
\end{aligned}
$$

Since

$$
\begin{gathered}
\int_{0}^{h \wedge \tau} \int_{0}^{\infty} e^{-\beta s}\left[v\left(X_{s^{-}}^{\hat{\theta}, \widehat{\gamma}}-Y, K_{s-}^{\hat{\theta}, \widehat{\gamma}}\right)-v\left(X_{s-}^{\hat{\theta}, \widehat{\gamma}}, K_{s-}^{\hat{\theta}, \widehat{\gamma}}\right)\right] \\
\cdot\left[\bar{K}(d Y, d s)-\lambda_{0} d G(Y) d s\right]
\end{gathered}
$$

is a martingale (see [28, page 63]), it follows that

$$
\begin{aligned}
& 0=\mathbb{E}\left[\int_{0}^{h \wedge \tau} e^{-\beta s}\left[-\beta v\left(X_{s}^{\hat{\theta}, \widehat{\gamma}}, K_{s}^{\hat{\theta}, \widehat{\gamma}}\right)+\mathscr{L}^{\hat{\theta}, \widehat{\gamma}} v\left(X_{s}^{\hat{\theta}, \widehat{\gamma}}, K_{s}^{\hat{\theta}, \widehat{\gamma}}\right)+U\left(\widehat{\gamma}_{s}\right)\right] d s\right]+\mathbb{E}\left[\int_{0}^{h \wedge \tau} e^{-\beta s}\left(-\lambda v_{x}+v_{k}\right)\left(X_{s}^{\widehat{\theta}, \widehat{\gamma}}, K_{s}^{\hat{\theta}, \widehat{\gamma}}\right) d M_{s}\right] \\
& +\lambda_{0} \mathbb{E}\left[\int_{0}^{h \wedge \tau} \int_{0}^{\infty} e^{-\beta s}\left[v\left(X_{s^{-}}^{\widehat{\theta}, \widehat{\gamma}}-Y, K_{s^{-}}^{\widehat{\theta}, \widehat{\gamma}}\right)-v\left(X_{s^{-}}^{\widehat{\theta}, \widehat{\gamma}}, K_{s^{-}}^{\widehat{\theta}, \widehat{\gamma}}\right)\right] d G(Y) d s\right] .
\end{aligned}
$$

With boundary condition (26), dividing proceeding equation by $h$ and sending $h$ to 0 , we can formally write the HJB function:

$$
\begin{aligned}
& \left(\lambda_{0}+\beta\right) v(x, k)-\sup _{(\theta, \gamma) \in \mathscr{A}(x, k)}\left\{U(\gamma)+\mathscr{L}^{\theta, \gamma} v(x, k)\right. \\
& \left.+\lambda_{0} \int_{0}^{x} v(x-Y, k) d G(Y)\right\}=0,
\end{aligned}
$$

for $x \geq 0, k>x$,

$$
\lambda v_{x}(x, x)-v_{k}(x, x)=0, \quad \text { for } x>0 .
$$

Boundary condition (41) comes from the fact that the wealth process will reflect whenever $X=K$ with direction $\tilde{\gamma}$ and the gradient of $v(x, k)$ is perpendicular to $\tilde{\gamma}$ at $x=k$.

If we can find a smooth solution for the HJB, then the optimal consumption will actually be given in feedback form by

$$
\widehat{\gamma}(x, k)=I\left(v_{x}(x, k)+v_{n}(x, k)\right),
$$

where $I \triangleq\left(U^{\prime}\right)^{-1}$ is the inverse of marginal utility. In addition, we expect the optimal amount invested in the fund to be given by

$$
\widehat{\theta}(x, k)=-\frac{\alpha}{\sigma^{2}} \cdot \frac{v_{x}(x, k)}{v_{x x}(x, k)} .
$$

Usually, it is difficult to justify the smoothness of value function or the existence of classical solution to the HJB equation that appeared in a control problem. The theory of viscosity principally provides us with a way to analyze our problem (cf. Crandall et al. [29]). To proceed our discussion, we need the following alternative expressions of dynamic programming principle; the readers are referred to Pham [30]. In the sequel, we denote by $\mathscr{T}_{0, \tau}$ the set of stopping times valued in $[0, \tau]$; then one has the following.

Proposition 5. (1) For all $(\theta, \gamma) \in \mathscr{A}(x, k)$ and $\vartheta \in \mathscr{T}_{0, \tau}$,

$$
v(x, k) \geq \mathbb{E}\left[\int_{0}^{\vartheta} e^{-\beta s} U\left(\gamma_{s}\right) d s+e^{-\beta \vartheta} v\left(X_{\vartheta}^{\theta, \gamma}, K_{\vartheta}^{\theta, \gamma}\right)\right] .
$$

(2) For all $\varepsilon>0$, there exists $(\theta, \gamma) \in \mathscr{A}(x, k)$ such that, for all $\vartheta \in \mathscr{T}_{0, \tau}$,

$$
\begin{aligned}
& v(x, k)-\varepsilon \\
& \quad \leq \mathbb{E}\left[\int_{0}^{\vartheta} e^{-\beta s} U\left(\gamma_{s}\right) d s+e^{-\beta \vartheta} v\left(X_{\vartheta}^{\theta, \gamma}, K_{\vartheta}^{\theta, \gamma}\right)\right] .
\end{aligned}
$$

\section{Value Function, Viscosity Solution, and Its Regularity}

4.1. Value Function and Viscosity Solution. In order to introduce the concept of viscosity solutions, we first introduce some additional notations. Given a locally bounded function $\omega$ (i.e., for all $(x, k) \in(0,+\infty) \times(0,+\infty)$, there exists a compact neighborhood $V$ of $(x, k)$ such that $\omega$ is bounded on $V$ ); we define its upper-semicontinuous envelope $\omega^{*}$ and lower-semicontinuous envelope $\omega_{*}$ on $[0,+\infty) \times(0,+\infty)$ by

$$
\begin{aligned}
& \omega^{*}(x, k)=\limsup _{\left(x^{\prime}, k^{\prime}\right) \rightarrow(x, k)} \omega\left(x^{\prime}, k^{\prime}\right), \\
& \omega_{*}(x, k)=\liminf _{\left(x^{\prime}, k^{\prime}\right) \rightarrow(x, k)} \omega\left(x^{\prime}, k^{\prime}\right) .
\end{aligned}
$$


Recall that $\omega^{*}$ (resp., $\omega_{*}$ ) is the smallest (resp., largest) upper-semicontinuous function (u.s.c.) above (resp., lowersemicontinuous function (l.s.c.) below) $\omega$ on $(0,+\infty) \times$ $(0,+\infty)$. Note that a locally bounded function $\omega$ on $(0,+\infty) \times(0,+\infty)$ is lower-semicontinuous (resp., uppersemicontinuous) if and only if $\omega=\omega_{*}$ on $(0,+\infty) \times(0,+\infty)$, and it is continuous if (and only if) $\omega=\omega^{*}=\omega_{*}$ on $(0,+\infty) \times(0,+\infty)$

Remark 6. Here, the first and second partial derivatives with respect to $x$ at $x=0$ mean the right partial derivatives.

Definition 7 (viscosity subsolution and supersolution). An u.s.c. function $\omega \in C$ is a viscosity subsolution of (40) iff for any test function $\psi \in C^{2,1}(0,+\infty) \times(0,+\infty)$; if $(\bar{x}, \bar{k})$ is a global maximum point of $\omega^{*}-\psi$, then

$$
\begin{aligned}
& \beta \psi(\bar{x}, \bar{k})-\sup _{(\theta, \gamma) \in \mathscr{A}(\bar{x}, \bar{k})}\left\{U(\gamma)+\mathscr{L}^{\theta, \gamma} \psi(\bar{x}, \bar{k})\right. \\
& \left.+\lambda_{0} \mathbb{E}[\psi(\bar{x}-Y, \bar{k})-\psi(\bar{x}, \bar{k})]\right\} \leq 0, \\
& \lambda \psi_{x}(\bar{x}, \bar{k})-\psi_{k}(\bar{x}, \bar{k}) \leq 0 .
\end{aligned}
$$

A l.s.c. function $\omega \in C$ is a viscosity supersolution of (40) iff for any test function $\varphi \in C^{2,1}((0,+\infty) \times(0,+\infty))$; if $(\bar{x}, \bar{k})$ is a global minimum point of $\omega_{*}-\psi$, then

$$
\begin{aligned}
& \beta \varphi(\bar{x}, \bar{k})-\sup _{(\theta, \gamma) \in \mathscr{A}(\bar{x}, \bar{k})}\left\{U(\gamma)+\mathscr{L}^{\theta, \gamma} \varphi(\bar{x}, \bar{k})\right. \\
& \left.+\lambda_{0} \mathbb{E}[\varphi(\bar{x}-Y, \bar{k})-\varphi(\bar{x}, \bar{k})]\right\} \geq 0, \\
& \lambda \varphi_{x}(\bar{x}, \bar{k})-\varphi_{k}(\bar{x}, \bar{k}) \geq 0 .
\end{aligned}
$$

Finally, $\omega$ is a viscosity solution of (40) if it is simultaneously a viscosity subsolution and supersolution.

In addition to Definition 7, there are three equivalent definitions on second-ordered Integro-differential partial differential equations; the readers who are interested in the proof of the equivalence of these definitions are referred to Benth et al. [31] or Barles and Imbert [32].

Theorem 8. $v(x, k)$ is a viscosity solution of $(40)$, where $v(x, k)$ was defined in (25).

Proof. Let us prove firstly that $v$ is a viscosity supersolution. Let $(\bar{x}, \bar{k}) \in(0,+\infty) \times(0,+\infty)$ and let $\varphi \in C^{2,1}((0,+\infty) \times$ $(0,+\infty))$ be a test function such that

$$
\begin{aligned}
0 & =\left(v_{*}-\varphi\right)(\bar{x}, \bar{k}) \\
& =\min _{(x, k) \in(0,+\infty) \times(0,+\infty)}\left(v_{*}-\varphi\right)(x, k) .
\end{aligned}
$$

We further extend the domain of $\varphi(x, k)$ to $\mathbb{R} \times(0,+\infty)$ with the convention that $\varphi(x, k)=0$ for all $x<0$. One will see later that such extension does not prevent us from discussing our problem. By definition of $v_{*}(\bar{x}, \bar{k})$, there exists a sequence $\left(x_{m}, k_{m}\right)$ in $(0,+\infty) \times(0,+\infty)$, such that

$$
\begin{gathered}
\left(x_{m}, k_{m}\right) \longrightarrow(\bar{x}, \bar{k}), \\
v\left(x_{m}, k_{m}\right) \longrightarrow v_{*}(\bar{x}, \bar{k}),
\end{gathered}
$$

when $m$ goes to infinity. By the continuity of $\varphi$ and by (49) we also have that

$$
\zeta_{m}:=v\left(x_{m}, k_{m}\right)-\varphi\left(x_{m}, k_{m}\right) \longrightarrow 0,
$$

when $m$ goes to infinity.

Let $(\theta, \gamma) \in \mathscr{A}(x, k)$; we denote by $\left(X_{s}^{\theta, \gamma}, K_{s}^{\theta, \gamma}\right)$ the associated controlled process. Let $\tau_{m}^{1}$ and $\tau_{m}^{2}$ be the stopping times given by $\tau_{m}^{1}=\inf \left\{0 \leq s \leq \tau:\left|X_{s}^{\theta, \gamma}\left(x_{m}, k_{m}\right)-x_{m}\right| \geq \eta\right\}$ and $\tau_{m}^{2}=\inf \left\{0 \leq s \leq \tau:\left|K_{s}^{\theta, \gamma}\left(x_{m}, k_{m}\right)-k_{m}\right| \geq \eta\right\}$ in which $\eta>0$ is a fixed constant, and $\tau_{m}:=\tau_{m}^{1} \wedge \tau_{m}^{2}$. Let $\left(h_{m}\right)$ be a strictly positive sequence such that

$$
\begin{aligned}
& h_{m} \longrightarrow 0, \\
& \frac{\zeta_{m}}{h_{m}} \longrightarrow 0
\end{aligned}
$$

when $m$ goes to infinity. We apply the first part of the dynamic programming principle (44) for $v\left(x_{m}, k_{m}\right)$ to $\vartheta_{m}:=\tau_{m} \wedge h_{m}$ and get

$$
\begin{aligned}
& v\left(x_{m}, k_{m}\right) \\
& \quad \geq \mathbb{E}\left[\int_{0}^{\vartheta_{m}} e^{-\beta s} U\left(\gamma_{s}\right) d s+e^{-\beta \vartheta_{m}} v\left(X_{\vartheta_{m}}^{\theta, \gamma}, K_{\vartheta_{m}}^{\theta, \gamma}\right)\right] .
\end{aligned}
$$

Equation (49) implies that $v \geq v_{*} \geq \varphi$. Thus

$$
\begin{aligned}
& \varphi\left(x_{m}, k_{m}\right)+\zeta_{m} \\
& \quad \geq \mathbb{E}\left[\int_{0}^{\vartheta_{m}} e^{-\beta s} U\left(\gamma_{s}\right) d s+e^{-\beta \vartheta_{m}} \varphi\left(X_{\vartheta_{m}}^{\theta, \gamma}, K_{\vartheta_{m}}^{\theta, \gamma}\right)\right] .
\end{aligned}
$$

Applying Itô's formula to $e^{-\beta \vartheta_{s}} \varphi\left(X_{\vartheta_{s}}^{\theta, \gamma}, K_{\vartheta_{s}}^{\theta, \gamma}\right)$ between 0 and $\vartheta_{m}$, we obtain

$$
\begin{aligned}
\frac{\zeta_{m}}{h_{m}}+ & \mathbb{E}\left[\frac { 1 } { h _ { m } } \int _ { 0 } ^ { \vartheta _ { m } } \left(\beta \varphi-U\left(\gamma_{s}\right)-\mathscr{L}^{\theta, \gamma} \varphi\right.\right. \\
& \left.\left.-\lambda_{0} \mathbb{E}[\varphi(X-Y, K)-\varphi(X, K)]\right)\left(X_{s}^{\theta, \gamma}, K_{s}^{\theta, \gamma}\right) d s\right] \\
& +\mathbb{E}\left[\frac{1}{h_{m}} \int_{0}^{\vartheta_{m}}\left(\lambda \varphi_{x}-\varphi_{k}\right)\left(X_{s}^{\theta, \gamma}, K_{s}^{\theta, \gamma}\right) d M_{s}\right] \geq 0,
\end{aligned}
$$

after noting that the stochastic integral term cancels out by taking expectations since the integrand is bounded. Since the random variable inside the expectation in (55) is bounded by a constant independent of $m$, we then obtain

$$
\begin{gathered}
\left(\lambda_{0}+\beta\right) \varphi(x, k)-U(\gamma)-\mathscr{L}^{\theta, \gamma} \varphi(x, k) \\
-\lambda_{0} \int_{0}^{x} \varphi(x-Y, k) d G(Y) \geq 0, \\
\lambda \varphi_{x}(x, k)-\varphi_{k}(x, k) \geq 0,
\end{gathered}
$$


when $m$ goes to infinity by the dominated convergence theorem. We conclude from the arbitrariness of $(\theta, \gamma) \in$ $\mathscr{A}(x, k)$. Thus we get (48).

It remains to prove that $v$ is a viscosity subsolution. Let $(\bar{x}, \bar{k}) \in(0,+\infty) \times(0,+\infty)$ and let $\psi \in C^{2,1}((0,+\infty) \times(0,+\infty))$ be a test function such that

$$
0=\left(v^{*}-\psi\right)(\bar{x}, \bar{k})=\max _{(x, k) \in R^{2}}\left(v^{*}-\psi\right)(x, k) .
$$

We will show the result by contradiction. Assume on the contrary that

$$
\begin{aligned}
& \beta \psi(\bar{x}, \bar{k})-\sup _{(\theta, \gamma) \in \mathscr{A}(x, k)}\left\{U(\gamma)+\mathscr{L}^{\theta, \gamma} \psi(\bar{x}, \bar{k})\right. \\
& \left.+\lambda_{0} \mathbb{E}[\psi(\bar{x}-Y, \bar{k})-\psi(\bar{x}, \bar{k})]\right\}>0, \\
& \lambda \psi_{x}(\bar{x}, \bar{k})-\psi_{k}(\bar{x}, \bar{k})>0 .
\end{aligned}
$$

There exist $\eta>0$ and $\varepsilon>0$ such that

$$
\begin{aligned}
& \beta \psi\left(x^{\prime}, k^{\prime}\right)-\sup _{(\theta, \gamma) \in \mathscr{A}(x, k)}\left\{U(\gamma)+\mathscr{L}^{\theta, \gamma} \psi\left(x^{\prime}, k^{\prime}\right)\right. \\
& \left.+\lambda_{0} \mathbb{E}\left[\psi\left(x^{\prime}-Y, k^{\prime}\right)-\psi\left(x^{\prime}, k^{\prime}\right)\right]\right\} \geq \varepsilon, \\
& \lambda \psi_{x}\left(x^{\prime}, k^{\prime}\right)-\psi_{n}\left(x^{\prime}, k^{\prime}\right) \geq \varepsilon,
\end{aligned}
$$

for all $\left(x^{\prime}, k^{\prime}\right) \in B(\bar{x}, \bar{k}, \eta)=\left\{\left(x^{\prime}, k^{\prime}\right) \in(0,+\infty) \times(0,+\infty)\right.$ : $\left.\left|\bar{x}-x^{\prime}\right|^{2}+\left|\bar{k}-k^{\prime}\right|^{2}<\eta\right\}$. By the definition of $v(\bar{x}, \bar{k})$, there exists a sequence $\left(x_{m}, k_{m}\right)$ taking values in $B(\bar{k}, \eta)$ such that

$$
\begin{gathered}
\left(x_{m}, k_{m}\right) \longrightarrow(\bar{x}, \bar{k}), \\
v\left(x_{m}, k_{m}\right) \longrightarrow v(\bar{x}, \bar{k}),
\end{gathered}
$$

when $m$ goes to infinity. By continuity of $\psi$ and using (57), we also find that

$$
\zeta_{m}:=v\left(x_{m}, k_{m}\right)-\psi\left(x_{m}, k_{m}\right) \longrightarrow 0,
$$

when $m$ goes to infinity. Let $\left(h_{m}\right)$ be a strictly positive sequence such that

$$
\begin{aligned}
& h_{m} \longrightarrow 0, \\
& \frac{\zeta_{m}}{h_{m}} \longrightarrow 0 .
\end{aligned}
$$

Then, according to the second part of dynamic programming principle (45) and using (57), there is a sequence $\left(\theta_{m}, \gamma_{m}\right) \in$ $\mathscr{A}\left(x_{m}, k_{m}\right)$ such that

$$
\begin{aligned}
& \psi\left(x_{m}, k_{m}\right)+\zeta_{m}-\frac{\varepsilon h_{m}}{2} \\
& \leq \mathbb{E}\left[\int_{0}^{\vartheta_{s}} e^{-\beta s} U\left(\gamma_{m}\right) d s+e^{-\beta \vartheta_{m}} \psi\left(X_{\vartheta_{m}}^{\theta_{m}, \gamma_{m}}, K_{\vartheta_{m}}^{\theta_{m}, \gamma_{m}}\right)\right],
\end{aligned}
$$

in which we take $\vartheta_{m}=\tau_{m}^{\prime} \wedge h_{m}, \tau_{m}^{\prime}=\tau_{m}^{3} \wedge \tau_{m}^{4}, \tau_{m}^{3}=\inf \{0 \leq$ $\left.s \leq \tau:\left|X_{s}^{\hat{\theta}, \hat{\gamma}}\left(x_{m}, k_{m}\right)-x_{m}\right| \geq \eta^{\prime}\right\}, \tau_{m}^{4}=\inf \{0 \leq s \leq \tau:$ $\left.\left|K_{s}^{\theta, \gamma}\left(x_{m}, k_{m}\right)-k_{m}\right| \geq \eta^{\prime}\right\}$, and $0<\eta^{\prime}<\eta$. Since $\left(x_{m}, k_{m}\right)$ converges to $(\bar{x}, \bar{k})$, we can always assume that $B\left(x_{m}, k_{m}, \eta^{\prime}\right) \subset$ $B(\bar{x}, \bar{k}, \eta)$. For $0 \leq s \leq \vartheta_{m} \leq \tau$, by applaying Itô's formula to $e^{-\beta s} \psi\left(X_{s}^{\theta_{m}, \gamma_{m}}, K_{s}^{\theta_{m}, \gamma_{m}}\right)$, we get

$$
\begin{array}{r}
0 \geq \frac{\zeta_{m}}{h_{m}}-\frac{\varepsilon}{2}+\mathbb{E}\left[\frac{1}{h_{m}} \int_{0}^{\vartheta_{m}} L\left(X_{s}^{\theta_{m}, \gamma_{m}}, K_{s}^{\theta_{m}, \gamma_{m}}\right) d s\right] \\
+\mathbb{E}\left[\frac{1}{h_{m}} \int_{0}^{\vartheta_{m}}\left(\lambda \psi_{x}-\psi_{k}\right)\left(X_{s}^{\theta, \gamma}, K_{s}^{\theta, \gamma}\right) d M_{s}\right]
\end{array}
$$

with

$$
\begin{aligned}
L(x, k)= & \beta v(x, k)-U(\gamma)-\mathscr{L}^{\theta, \gamma} \psi(x, k) \\
& -\lambda_{0} \mathbb{E}[\psi(x-Y, k)-\psi(x, k)],
\end{aligned}
$$

after noting that the stochastic integral term cancels out by taking expectations since the integrand is bounded.

Moreover, noting that for $0 \leq s<\vartheta_{m} \leq \tau$

$$
\begin{aligned}
& L\left(X_{s}^{\theta_{m}, \gamma_{m}}, K_{s}^{\theta_{m}, \gamma_{m}}\right) \geq \beta v(x, k)-\sup _{(\theta, \gamma) \in \mathscr{A}(x, k)}\{U(\gamma) \\
& \left.\quad+\mathscr{L}^{\theta, \gamma} \psi(x, k)+\lambda_{0} \mathbb{E}[\psi(x-Y, k)-\psi(x, k)]\right\} \\
& \quad \geq \varepsilon
\end{aligned}
$$

we find using (59) and (64) that

$$
0 \geq \frac{\zeta_{m}}{h_{m}}-\varepsilon\left(\frac{1}{2}-\frac{1}{h_{m}} \mathbb{E}\left[\vartheta_{m}\right]\right)
$$

since (see Pham [30, Page 38])

$$
\begin{aligned}
& \lim _{h_{m \downarrow 0^{+}}} \mathbb{E}\left[\sup _{s \in\left(0,9_{m}\right]}\left|X_{s}^{\widehat{\Theta}_{m}, \widehat{\gamma}_{m}}-x_{m}\right|^{2}\right]=0, \\
& \lim _{h_{m \downarrow 0^{+}}} \mathbb{E}\left[\sup _{s \in\left(0,9_{m}\right]}\left|K_{s}^{\widehat{\theta}_{m}, \widehat{\gamma}_{m}}-k_{m}\right|^{2}\right]=0 .
\end{aligned}
$$

By Chebyshev's inequality, we deduce that

$$
\begin{aligned}
\mathbb{P}\left[\tau_{m}^{\prime} \leq h_{m}\right] \leq & \mathbb{P}\left[\sup _{s \in\left(0, h_{m}\right]}\left|X_{s}^{\hat{\theta}_{m}, \widehat{\gamma}_{m}}-x_{m}\right| \geq \eta\right] \\
& \cdot \mathbb{P}\left[\sup _{s \in\left(0, h_{m}\right]}\left|K_{s}^{\widehat{\theta}_{m}, \widehat{\gamma}_{m}}-k_{m}\right| \geq \eta\right] \\
\leq & \frac{\mathbb{E}\left|\sup _{s \in\left(0, h_{m}\right]}\right| X_{s}^{\widehat{\theta}_{m}, \widehat{\gamma}_{m}}-\left.x_{m}\right|^{2} \mid}{\eta^{2}} \\
& \frac{\mathbb{E}\left|\sup _{s \in\left(0, h_{m}\right]}\right| K_{s}^{\widehat{\theta}_{m}, \widehat{\gamma}_{m}}-\left.k_{m}\right|^{2} \mid}{\eta^{2}} \longrightarrow 0,
\end{aligned}
$$


when $h_{m}$ goes to zero, that is, when $m$ goes to infinity. Moreover, since

$$
\mathbb{E}\left[\vartheta_{t}\right]=\int_{\left\{\tau_{m}^{\prime}>h_{m}\right\}} h_{m} d \mathbb{P}+\int_{\left\{\tau_{m}^{\prime} \leq h_{m}\right\}}\left(\tau_{m}^{\prime}\right) d \mathbb{P}
$$

we deduce that

$$
\begin{aligned}
h_{m} \mathbb{P}\left(\tau_{m}^{\prime}>h_{m}\right) & =h_{m} \mathbb{P}\left(\tau_{m}^{\prime}>h_{m}\right)=\int_{\left\{\tau_{m}^{\prime}>h_{m}\right\}} h_{m} d \mathbb{P} \\
& \leq \mathbb{E}\left[\vartheta_{t}\right] \\
& \leq \int_{\left\{\tau_{m}^{\prime}>h_{m}\right\}} h_{m} d \mathbb{P}+\int_{\left\{\tau_{m}^{\prime} \leq h_{m}\right\}} h_{m} d \mathbb{P} \\
& =h_{m} .
\end{aligned}
$$

So we obtain

$$
\mathbb{P}\left[\tau_{m}^{\prime}>h_{m}\right] \leq \frac{1}{h_{m}} \mathbb{E}\left[\vartheta_{m}\right] \leq 1 .
$$

This implies that $\left(1 / h_{m}\right) \mathbb{E}\left[\vartheta_{m}\right]$ converges to 1 when $h_{m}$ goes to zero. We thus get the desired contradiction by letting $m$ go to infinity in (67).

So (47) holds and we complete the proof.

4.2. Dimension Reduction and Regularity of Viscosity Solution. A key insight noted by Magill and Constantinides [33] and exploited in Davis and Norman [8] is that because of the homotheticity of power utility function (Proposition 3.3) the dimension of our control problem is ready to be reduced from two to one. In Janeček and Sîrbu [9], where the decision maker is assumed to be a hedge fund manager, such reduction is successful and with such reduction, the authors proved the regularity of the viscosity solution to the HJB equation associated with their control problem. In our problem we guess that the value function, also the viscosity solution to the HJB equation, resembles similar property. The following intuitive interpretation will help us to understand this point. In Section 2.1, it has been shown that the ruin probability of classical risk model can be approximated to a drifted Brownian motion with proper drift and diffusion coefficients. What is more, one can even try to approximate the distribution of the functional of the maximum process of classical risk model by diffusion process. So, if we replace the classical risk model by a proper drifted Brownian motion, then after some easy calculations, one can find that the corresponding HJB equation shares the same formulation with the one presented in Janeček and Sîrbu [9]. In this situation, it is natural to guess that the value function can be reduced from two to one. The main difference of the HJB equation of this paper is that there is an integral term in the HJB equation, however, after noting that the control process is stopped after stopping time $\tau^{\theta, \gamma}$, so we still hope that there is a possibility to reduce the viscosity solution from two to one. More precisely, we expect that

$$
v(x, k)=x^{1-p} v\left(1, \frac{k}{x}\right) \triangleq x^{1-p} u(z) \quad \text { for } z \triangleq \frac{k}{x} .
$$

In addition, instead of looking for the optimal amounts $\widehat{\theta}(x, k)$ and $\widehat{\gamma}(x, k)$ in (43) and (42) we look for the proportions

$$
\begin{aligned}
& \widehat{c}(x, k)=\frac{\widehat{\gamma}}{x}=\frac{I\left(v_{x}(x, k)+v_{k}(x, k)\right)}{x}, \\
& \widehat{\pi}(x, k)=\frac{\widehat{\theta}}{x}=-\frac{\alpha}{\sigma^{2}} \cdot \frac{x v_{x}(x, k)}{x^{2} v_{x x}(x, k)} .
\end{aligned}
$$

Since

$$
\begin{aligned}
& v_{k}(x, k)=u^{\prime}(z) \cdot x^{-p}, \\
& v_{x}(x, k)=\left((1-p) u(z)-z u^{\prime}(z)\right) \cdot x^{-p}, \\
& v_{x x}(x, k) \\
& =\left(-p(1-p) u(z)+2 p z u^{\prime}(z)+z^{2} u^{\prime \prime}(z)\right) \\
& \quad \cdot x^{-1-p},
\end{aligned}
$$

it is followed that (40) and (41) can be reformulated as

$$
\begin{aligned}
& \sup _{\gamma>0, \theta}\left\{-\beta u+\frac{c^{1-p}}{1-p}+(\pi \alpha-c)\left[(1-p) u-z u^{\prime}\right]-c u^{\prime}\right. \\
& \quad+\frac{1}{2} \pi^{2} \sigma^{2}\left(-p(1-p) u+2 p z u^{\prime}+z^{2} u^{\prime \prime}\right) \\
& \left.\quad+\lambda_{0} \chi(u(z))\right\}=0 \\
& -\lambda(1-p) u(1)+(1+\lambda) u^{\prime}(1)=0
\end{aligned}
$$

where, for notation simplicity, we adopt $\chi(u(z))$ for $\int_{0}^{x}(x-$ $Y, k) d G(y)$. We also expect that

$$
\lim _{z \rightarrow \infty} u(z)=\frac{1}{1-p} c_{0}^{-p}
$$

with $c_{0}$ given by (96) below; see (98).

The optimal investment proportion in (75) could therefore be expressed (if we can find a smooth solution for reduced $\mathrm{HJB}(77)$ ) as

$$
\widehat{\pi}(z)=\frac{\alpha}{p \sigma^{2}} \cdot \frac{(1-p) u-z u^{\prime}}{(1-p) u+2 z u^{\prime}-(1 / p) z^{2} u^{\prime \prime}},
$$

and the optimal consumption proportion $\widehat{c}$ in (74) would be given by

$$
\widehat{c}(z)=\frac{\left(v_{x}+v_{k}\right)^{-1 / p}}{x}=\left((1-p) u-(z-1) u^{\prime}\right)^{-1 / p} .
$$

The following theorem asserts the regularity of the viscosity solution to (77) with boundary condition (78). 
Theorem 9. The function $u$ is $C^{2}$ on $[1, \infty)$ and satisfies

$$
\begin{array}{r}
-p(1-p) u+2 p z u^{\prime}+z^{2} u^{\prime \prime}<0, \\
(1-p) u-(z-1) u^{\prime}>0, \\
(1-p) u-z u^{\prime}>0 .
\end{array}
$$

$z>1$.

Moreover, it is a solution of the equation

$$
\begin{aligned}
\sup _{c \geq 0, \pi} \mathscr{L}_{c, \pi} u & \\
= & -\beta u+\widetilde{V}\left((1-p) u-(z-1) u^{\prime}\right)+\lambda_{0} \chi(u) \\
& \quad-\frac{1}{2} \frac{\alpha^{2}}{\sigma^{2}} \frac{\left((1-p) u-z u^{\prime}\right)^{2}}{-p(1-p) u+2 p z u^{\prime}+z^{2} u^{\prime \prime}}, \quad z>1, \\
-\lambda(1-p) u(1)+(1+\lambda) u^{\prime}(1)=0, &
\end{aligned}
$$

where

$$
\begin{aligned}
& \widetilde{V}(y)=\left\{\begin{array}{ll}
\frac{p}{1-p} y^{(p-1) / p}, & y>0, \\
+\infty, & y \leq 0,
\end{array} \text { for } p<1,\right. \\
& \widetilde{V}(y)=\left\{\begin{array}{ll}
\frac{p}{1-p} y^{(p-1) / p}, & y \geq 0, \\
+\infty, & y<0,
\end{array} \text { for } p>1 .\right.
\end{aligned}
$$

Proof. The proof is very similar to the one for Theorem 5.2 of Janeček and Sîrbu [9] more or less; we do not copy the steps here. One just needs to note that the HJB equation in this paper differs from the one in Janeček and Sîrbu [9] lies in $\chi(u)$; however, this term is not involved in the discussion of the regularity of viscosity.

Remark 10. Although the jump term of insurer does not affect the smoothness of the value function of our control problem, due to the existence of such jump term, the value function and consequently the optimal policies will be highly influenced. This will be illustrated in the next section by partial analysis on the properties to the viscosity solution.

Theorem 9 claims the regularity of value function $v(x, k)$ when $x>0$. When $x=0$, the value function $v(0, k)$ is specified by the following theorem.

Theorem 11. $v(0, k)$ satisfies

$$
\begin{aligned}
& \beta v(0, k) \\
& \quad-\sup _{0 \leq \gamma \leq a}\left\{U(\gamma)+(a-\gamma) v_{0}(0, k)-\gamma v_{k}(0, k)\right\} \\
& =0 .
\end{aligned}
$$

Proof. If initial surplus of insurer $x=0$, then to invest any amount on risky market can be optimal since the diffusion property of the risky market will cause ruin to happen immediately (cf. Dufresne and Gerber [34]). So optimal investment for insurer is to invest 0 amount on risky market in a very small interval, and of course, the optimal consumption rate $\gamma_{t}$, which is to be determined, should not exceed the premium income rate, say $a$. Based on this analysis, the HJB function for value function at $x=0$ is reduced to

$$
\begin{aligned}
& \beta v(0, k) \\
& \quad-\sup _{0 \leq \gamma \leq a}\left\{U(\gamma)+(a-\gamma) v_{0}(0, k)-\gamma v_{k}(0, k)\right\}
\end{aligned}
$$

$$
=0 \text {. }
$$

4.3. Asymptotic Properties of Value Function. In this section, we will have some asymptotic properties of value function.

Lemma 12. $v(x, k)$ is bounded on $[0, \infty) \times(0, \infty)$.

Proof. Revisit the definition of $v(x, k)$, suppose that at time $t$ the wealth process of insurer is $X_{t}^{\theta, \gamma}$, and then obviously $\gamma_{t} \leq X_{t}^{\theta, \gamma}$, or else the ruin will take place, which cannot be the optimal policy for insurer. Thus, one can see that

$$
v(x, k) \leq \mathbb{E}\left[\int_{0}^{\tau} e^{-\beta t} U\left(\widetilde{X}_{t}^{\theta, 0}\right)\right],
$$

where the wealth process $\widetilde{X}_{t}, 0 \leq t \leq \tau$, is the one under policy $\theta$ and $\gamma_{t} \equiv 0$. So, the policy that maximizes the ruin time $\tau$ will maximize $\mathbb{E}\left[\int_{0}^{\tau} e^{-\beta t} U\left(\widetilde{X}_{t}^{\theta, 0}\right)\right]$. Yang and Zhang [35] prove that a constant investment policy maximizes this amount. If the insurer adopts the constant investment policy, then the wealth process of insurer is

$$
X_{t}=x+a t-Z_{t}+C^{*} *\left(\alpha t+\sigma W_{t}\right), \quad t \geq 0,
$$

where $C^{*}$ is the constant investment policy. Then, if $p \leq 1$, it is easy to see that $\mathbb{E}\left[\int_{0}^{\tau} e^{-\beta t} U\left(\widetilde{X}_{t}^{C^{*}, 0}\right)\right]$ is bounded. If $p>1$, Protter [36] shows that

$$
\mathbb{E}\left[\sup _{0 \leq s \leq t}\left|X_{s}^{C^{*}, 0}\right|^{p}\right] \leq \Gamma e^{\rho t}\left(1+x^{p}\right),
$$

where $\Gamma$ and $\rho$ are constants depending on coefficients involved in the wealth process. Thus, by choosing a large enough $\beta$, it follows that

$$
\mathbb{E}\left[\int_{0}^{\tau} e^{-\beta t} U\left(\widetilde{X}_{t}^{C^{*}, 0}\right)\right]<\Gamma \int_{0}^{\infty} e^{-(\beta-\rho) t} x^{1-p} d t .
$$

This indicates that $v(x, k)$ is bounded.

Theorem 13. For $\hat{\pi}$ and $\widehat{c}$ that are defined by (74) and (75), one has

$$
\begin{aligned}
\lim _{z \rightarrow \infty} \widehat{\pi}(z) & =\frac{\alpha}{p \sigma^{2}}, \\
\lim _{z \rightarrow \infty} \widehat{c}(z) & =((1-p) u(\infty))^{-1 / p},
\end{aligned}
$$


and $u(\infty) \triangleq \lim _{z \rightarrow \infty} u(z)$ is determined by

$$
\beta u(\infty)-\chi(u(\infty))=\widetilde{V}((1-p) u(\infty)),
$$

where $\chi(u(\infty))=\lim _{z \rightarrow \infty} \int_{0}^{1} v(x-Y, k) d G(y)=$ $u(\infty) \int_{0}^{1}(x-Y)^{1-p} d G(y)$.

Proof. Note that $u(z) \in C^{2}[1, \infty)$ and $u(z)=v(1, z)$, by Theorem 9 one can prove that

$$
\begin{array}{r}
z u^{\prime}(z) \longrightarrow 0, \\
z^{2} u^{\prime \prime}(z) \longrightarrow 0,
\end{array}
$$

$$
z \longrightarrow \infty \text {. }
$$

Here we assume that the above limits exist; in fact, by repeating a similar discussion to the proof for Proposition 4.1 of Janeček and Sîrbu [9], such assumptions are guaranteed. By (80) and (81) and (94), we have (92) immediately. Let $z \rightarrow \infty$ in (83), and by (94) we have (93).

Remark 14. (1) (the case when paying no fee $\lambda=0$ and $\left.\lambda_{0}=0\right)$ This is the classical problem in Merton [2,3] and can be solved in closed form. More precisely, for $\lambda=0$, the optimal investment and consumption proportions are constant, which are given by

$$
\begin{aligned}
& \pi_{0} \triangleq \frac{\alpha}{p \sigma^{2}} \\
& c_{0} \triangleq \frac{\beta}{p}-\frac{1}{2} \frac{1-p}{p^{2}} \cdot \frac{\alpha^{2}}{\sigma^{2}} .
\end{aligned}
$$

The Merton value function (and solution of the HJB) equals

$$
v_{0}(x, k)=\frac{1}{1-p} c_{0}^{-p} x^{1-p}, \quad 0<x \leq n .
$$

It follows that for $\lambda=0$

$$
u_{0}(z)=\frac{1}{1-p_{0}} c_{0}^{-p}, \quad z \geq 1
$$

Since $u_{0}$ in (98) is constant, (95) and (96) are compatible with the feedback formulas (80) and (81).

As can be easily seen from above, for the case $0<p<$ 1 , in order to obtain a finite value function, an additional constraint needs to be imposed on the parameters. This is equivalent to $c_{0}$ in (96) being strictly positive, which translates to the standing assumption

$$
\beta>\frac{1}{2} \frac{1-p}{p} \cdot \frac{\alpha^{2}}{\sigma^{2}}, \quad \text { if } 0<p<1 .
$$

(2) When $\lambda=0$, our model reduces to the case that an insurer would like to maximize his expected cumulative discount utility form consumption. To the best of our knowledge, this problem has not been addressed before. One may find that when $\lambda=0$, it means that the insurer does not need to pay any high-watermark fee for the gain profit, which is equal to the case that the initial high-watermark of the insurer is infinity in the model studied in this paper. Denote by $m(x) \triangleq \sup _{\theta, \gamma>0} \mathbb{E}^{x}\left[\int_{0}^{\tau} U\left(\gamma_{t}\right)\right]$ the value function of the insurer who does not need to be subject to high-watermark fee; then

$$
m(x)=v(x, \infty)=x^{1-p} v(1, \infty)=x^{1-p} u(\infty),
$$

where $u(\infty)$ is specified by (93). This is also the value function for the insurer without high-watermark fee.

Comparing $v_{0} x$ and $m(x)$, it is obvious that two functions share the same power formulation and differ on the constant term. These results indicate that there is no significant difference between the investment and consumption behavior between an insurance company and a hedge fund manager. This is not the first time that we observe such phenomenon; in fact, when we consider the optimal investment for maximizing the survival probability of an insurer (cf. Yang and Zhang [35]) or the one of a fund manager (cf. Browne [37]), the value function shares the same exponential form, which just differs on the constant term.

\section{The Verification Theorem}

Theorem 15 (the verification theorem). Let $\omega(x, k)$ be a function in $C^{2,1}((0,+\infty) \times(0, \infty))$ and satisfy a quadratic growth condition; that is, there exists a constant $D$ such that

$$
|\omega(x, k)| \leq D\left(1+|x|^{2}+|k|^{2}\right) \text {. }
$$

(1) Suppose that

$$
\begin{aligned}
& \beta \omega(x, k)-\sup _{(\theta, \gamma) \in \mathscr{A}(x, k)}\left\{U(\gamma)+\mathscr{L}^{\theta, \gamma} \omega(x, k)\right. \\
& \left.+\lambda_{0} \int_{0}^{x} \omega(x-Y, k) d G(Y)\right\} \geq 0, \\
& \forall(x, k) \in(0,+\infty] \times(0, \infty), \\
& \lambda \omega_{x}(x, k)-\omega_{n}(x, n) \geq 0, \\
& \limsup _{t \rightarrow \tau} e^{-\beta t} \mathbb{E}\left[\omega\left(X_{t}^{\theta, \gamma}, K_{t}^{\theta, \gamma}\right)\right] \geq 0, \\
& \quad(x, k) \in(0,+\infty) \times(0, \infty) .
\end{aligned}
$$

Then $\omega \geq v$ on $R^{2}$.

(2) Suppose further that, for all $(x, k) \in(0,+\infty) \times(0, \infty)$, there exists a measurable function $(\widehat{\theta}(x, k), \widehat{\gamma}(x, k)),(x, k) \in$ $(0,+\infty) \times(0, \infty)$, value in $\mathscr{A}$ such that

$$
\begin{aligned}
& \beta \omega(x, n)-\sup _{(\theta, \gamma) \in \mathscr{A}(x, k)}\left\{U(\gamma)+\mathscr{L}^{\theta, \gamma} \omega(x, k)\right. \\
& \left.+\lambda_{0} \int_{0}^{x} \omega(x-Y, k) d G(Y)\right\}=-\omega_{t}(x, k) \\
& -U(\widehat{\gamma})-\mathscr{L}^{\hat{\theta}, \hat{\gamma}} \omega(x, k)-\lambda_{0} \int_{0}^{x} \omega(x \\
& -Y, k) d G(Y)=0, \\
& \lambda \omega_{x}(x, k)-\omega_{n}(x, k)=0,
\end{aligned}
$$


and SDE (20) admits a unique solution, denoted by $\left(X_{s}^{\widehat{\theta}, \widehat{\gamma}}\right.$, $\left.K_{s}^{\hat{\theta}, \widehat{\gamma}}\right)$, given an initial condition $X_{0}=x$, which satisfies

$$
\liminf _{t \rightarrow \tau} e^{-\beta t} \mathbb{E}\left[\omega\left(X_{t}^{\theta, \gamma}, K_{t}^{\theta, \gamma}\right)\right] \leq 0,
$$

and the process $\left\{\left(\widehat{\theta}\left(X_{s}^{\widehat{\theta}, \widehat{\gamma}}, K_{s}^{\widehat{\theta}, \widehat{\gamma}}\right), \widehat{\theta}\left(X_{s}^{\widehat{\theta}, \widehat{\gamma}}, K_{s}^{\widehat{\theta}, \widehat{\gamma}}\right)\right)\right\}$ that stops at $\tau^{\theta, \gamma}$ lies in $\mathscr{A}(x, n)$.
Then

$$
\omega=v, \quad \text { on }(0, \infty) \times(0, \infty),
$$

and $(\widehat{\theta}, \widehat{\gamma})$ is an optimal Markovian control.

Proof. (1) Since $\omega \in C^{2,1}((0, \infty) \times(0, \infty))$, we have for all $(x, k) \in(0, \infty) \times(0, \infty),(\theta, \gamma) \in \mathscr{A}(x, k), s \in(0, \tau]$, similar to (37); by Itô formula and taking the expectation, we have

$$
\begin{aligned}
\mathbb{E}\left[e^{-\beta(t \wedge \tau)} \omega\left(X_{t \wedge \tau}^{\theta, \gamma}, K_{t \wedge \tau}^{\theta, \gamma}\right)\right]= & \omega(x, k)+\mathbb{E}\left[\int_{0}^{t \wedge \tau} e^{-\beta u}\left(-\beta \omega\left(X_{u}^{\theta, \gamma}, K_{u}^{\theta, \gamma}\right)+\mathscr{L}^{\theta, \gamma} \omega\left(X_{u}^{\theta, \gamma}, K_{u}^{\theta, \gamma}\right)\right) d u\right] \\
& +\mathbb{E}\left[\lambda_{0} \int_{0}^{t \wedge \tau} \int_{0}^{\infty} e^{-\beta u}\left[\omega\left(X_{u-}^{\theta, \gamma}-Y, K_{u-}^{\theta, \gamma}\right)-\omega\left(X_{u-}^{\theta, \gamma}, K_{u-}^{\theta, \gamma}\right)\right] d G(Y) d u\right] \\
& +\mathbb{E} \int_{0}^{t \wedge \tau} e^{-\beta u}\left(-\lambda \omega_{x}+\omega_{k}\right)\left(X_{u}^{\theta, \gamma}, K_{u}^{\theta, \gamma}\right) d M_{u} .
\end{aligned}
$$

Since $\omega$ satisfies (102), we have

$$
\begin{array}{r}
-\beta \omega\left(X_{u}^{\theta, \gamma}, K_{u}^{\theta, \gamma}\right)+U(\gamma)+\mathscr{L}^{\theta, \gamma} \omega\left(X_{u}^{\theta, \gamma}, K_{u}^{\theta, \gamma}\right) \\
+\lambda_{0} \mathbb{E}[\omega(X-Y, K)-\omega(X, K)] \leq 0, \\
\forall(\theta, \gamma) \in \mathscr{A}(x, k),
\end{array}
$$

$\lambda \omega_{x}(x, k)-\omega_{k}(x, k) \geq 0$

and so

$$
\begin{array}{r}
\mathbb{E}\left[e^{-\beta(T \wedge \tau)} \omega\left(X_{T \wedge \tau}^{\theta, \gamma}, K_{T \wedge \tau}^{\theta, \gamma}\right)\right] \\
\leq \omega(x, k)-\mathbb{E}\left[\int_{0}^{T \wedge \tau} e^{-\beta u} U\left(\gamma_{u}\right) d u\right], \\
\forall(\theta, \gamma) \in \mathscr{A}(x, k) .
\end{array}
$$

We have

$$
\left|\mathbb{E}\left[\int_{0}^{T \wedge \tau} e^{-\beta u} U\left(\gamma_{u}\right) d u\right]\right| \leq \int_{0}^{T \wedge \tau}\left|e^{-\beta u} U\left(\gamma_{u}\right)\right| d u .
$$

The right hand side of (111) is integrable by the integrability condition on $\mathscr{A}(x, k)$. According to (110), by sending $t$ to $\tau$, since $\omega$ satisfies a quadratic grown condition, we obtain by the dominated convergence theorem and by (104)

$$
\begin{aligned}
& 0 \leq \omega(x, k)-\mathbb{E}\left[\int_{0}^{T \wedge \tau} e^{-\beta u} U\left(\gamma_{u}\right) d u\right] \\
& \forall(\theta, \gamma) \in \mathscr{A}(x, k) .
\end{aligned}
$$

Since $(\theta, \gamma) \in \mathscr{A}(x, k)$ is arbitrary, we conclude that $\omega(x, k) \geq$ $v(x, k)$, for all $(x, k) \in R^{2}$.

(2) We apply Itô's formula to $e^{-\beta u} \omega\left(X_{u}^{\widehat{\theta}, \widehat{\gamma}}, K_{u}^{\widehat{\theta}, \widehat{\gamma}}\right)$ between 0 and $t$ (after an eventual localization for removing the stochastic integral term in the expectation):

$$
\begin{aligned}
\mathbb{E}\left[e^{-\beta t} \omega\left(X_{t}^{\hat{\theta}, \widehat{\gamma}}, K_{t}^{\widehat{\theta}, \widehat{\gamma}}\right)\right]= & \omega(x, k)+\mathbb{E}\left[\int_{0}^{T}\left(-\beta \omega\left(X_{u}^{\widehat{\theta}, \widehat{\gamma}}, K_{u}^{\widehat{\theta}, \widehat{\gamma}}\right)+\mathscr{L}^{\hat{\theta}, \hat{\gamma}} \omega\left(X_{u}^{\widehat{\theta}, \widehat{\gamma}}, K_{u}^{\widehat{\theta}, \widehat{\gamma}}\right)\right) d u\right] \\
& +\mathbb{E}\left[\lambda_{0} \int_{0}^{T} \int_{0}^{\infty} e^{-\beta u}\left[\omega\left(X_{u-}^{\widehat{\theta}, \widehat{\gamma}}-Y, K_{u-}^{\widehat{\theta}, \widehat{\gamma}}\right)-\omega\left(X_{u-}^{\widehat{\theta}, \widehat{\gamma}}, K_{u-}^{\widehat{\theta}, \widehat{\gamma}}\right)\right] d G(Y) d u\right] \\
& +\mathbb{E} \int_{0}^{t} e^{-\beta u}\left(-\lambda \omega_{x}+\omega_{k}\right)\left(u, X_{u}^{\hat{\theta}, \widehat{\gamma}}, K_{u}^{\hat{\theta}, \widehat{\gamma}}\right) d M_{u} .
\end{aligned}
$$

Now, by definition of $(\widehat{\theta}, \widehat{\gamma})$, we have

$$
\begin{aligned}
& \beta \omega(x, k)-U(\widehat{\gamma})-\mathscr{L}^{\widehat{\theta}, \widehat{\gamma}} \omega(x, k) \\
& \quad-\lambda_{0} \mathbb{E}[\omega(x-Y, K)-\omega(x, k)]=0, \\
& \lambda \omega_{x}(x, k)-\omega_{k}(x, k)=0,
\end{aligned}
$$

and so

$$
\begin{aligned}
\mathbb{E} & {\left[e^{-\beta t} \omega\left(X_{t}^{\hat{\theta}, \hat{\gamma}}, K_{t}^{\hat{\theta}, \hat{\gamma}}\right)\right] } \\
& =\omega(x, k)-\mathbb{E}\left[\int_{0}^{t} e^{-\beta u} U\left(\widehat{\gamma}_{u}\right) d u\right] .
\end{aligned}
$$


By sending $T$ to $\tau$ and from (106), we obtain

$$
\omega(x, k)=\mathbb{E}\left[\int_{0}^{\tau} e^{-\beta u} U\left(\widehat{\gamma}_{u}\right) d u\right] \leq v(x, k),
$$

and finally we obtain that $\omega=v$ with $(\widehat{\theta}, \widehat{\gamma})$ as an optimal Markovian control. So we complete the proof.

\section{Conclusions}

In this paper, we study the optimal investment and consumption problem of an insurer, where the consumption of insurer can be regarded as a kind of dividend payment. Thus, the problem considered in this paper is of practical relevance and reasonable. By dynamic programming method, the associated HJB equation is derived and the value function is proved to be the viscosity solutions. This result enables us to apply the numerical scheme for PDE, especially for HJB equation in viscosity sense (cf. Soner [38]) to find the optimal investment and consumption policies and the value function.

\section{Conflict of Interests}

The authors declare that there is no conflict of interests regarding the publication of this paper.

\section{Acknowledgments}

The authors are very grateful to editors and anonymous advices and help, which improved this paper greatly. This paper was supported by Natural Science Foundation of China (11201006 and 11301303), Humanities and Social Sciences Project of the Ministry of Education of China (12YJC910012, 14YJA630088, and 15YJC910008), and Natural Science Foundation of the Jiangsu Higher Education Institutions of China (15KJB110009).

\section{References}

[1] S. E. Shreve and H. M. Soner, "Optimal investment and consumption with transaction costs," The Annals of Applied Probability, vol. 4, no. 3, pp. 609-692, 1994.

[2] R. C. Merton, "Lifetime portfolio selection under uncertainty: the continuous-time case," The Review of Economics and Statistics, vol. 51, no. 3, pp. 247-257, 1969.

[3] R. C. Merton, "Optimum consumption and portfolio rules in a continuous-time model," Journal of Economic Theory, vol. 3, no. 4, pp. 373-413, 1971.

[4] T. Zariphopoulou, "Optimal investment and consumption models with non-linear stock dynamics," Mathematical Methods of Operations Research, vol. 50, no. 2, pp. 271-296, 1999.

[5] B. Øksendal and A. Sulem, "Optimal consumption and portfolio with both fixed and proportional transaction costs," SIAM Journal on Control and Optimization, vol. 40, no. 6, pp. 17651790, 2002.

[6] W. H. Fleming and T. Pang, "A stochastic control model of investment, production and consumption," Quarterly of Applied Mathematics, vol. 63, no. 1, pp. 71-87, 2005.

[7] I. Karatzas and S. E. Shreve, Methods of Mathematical Finance, vol. 39, Springer, 1998.
[8] M. H. Davis and A. R. Norman, "Portfolio selection with transaction costs," Mathematics of Operations Research, vol. 15, no. 4, pp. 676-713, 1990.

[9] K. Janeček and M. Sîrbu, "Optimal investment with highwatermark performance fee," SIAM Journal on Control and Optimization, vol. 50, no. 2, pp. 790-819, 2012.

[10] A. E. Whalley and P. Wilmott, "An asymptotic analysis of an optimal hedging model for option pricing with transaction costs," Mathematical Finance, vol. 7, no. 3, pp. 307-324, 1997.

[11] J. Cvitanić and I. Karatzas, "Hedging and portfolio optimization under transaction costs: a martingale approach," Mathematical Finance, vol. 6, no. 2, pp. 133-165, 1996.

[12] H. Liu and M. Loewenstein, "Optimal portfolio selection with transaction costs and finite horizons," Review of Financial Studies, vol. 15, no. 3, pp. 805-835, 2002.

[13] R. Korn, "Portfolio optimisation with strictly positive transaction costs and impulse control," Finance and Stochastics, vol. 2, no. 2, pp. 85-114, 1998.

[14] A. A. Obizhaeva and J. Wang, "Optimal trading strategy and supply/demand dynamics," Journal of Financial Markets, vol. 16, no. 1, pp. 1-32, 2013.

[15] J. E. Stiglitz, "Some aspects of the taxation of capital gains," Journal of Public Economics, vol. 21, no. 2, pp. 257-294, 1983.

[16] R. M. Dammon, C. S. Spatt, and H. H. Zhang, "Optimal consumption and investment with capital gains taxes," Review of Financial Studies, vol. 14, no. 3, pp. 583-616, 2001.

[17] W. N. Goetzmann, J. E. Ingersoll Jr., and S. A. Ross, "High-water marks and hedge fund management contracts," The Journal of Finance, vol. 58, no. 4, pp. 1685-1718, 2003.

[18] P. Guasoni and G. Wang, "High-water marks and separation of private investments," SSRN Electronic Journal, 2012.

[19] B. Højgaard and M. Taksar, "Optimal proportional reinsurance policies for diffusion models with transaction costs," Insurance: Mathematics and Economics, vol. 22, no. 1, pp. 41-51, 1998.

[20] A. Cairns, "Some notes on the dynamics and optimal control of stochastic pension fund models in continuous time," ASTIN Bulletin, vol. 30, no. 1, pp. 19-56, 2000.

[21] J. Zhu, "Optimal dividend control for a generalized risk model with investment incomes and debit interest," Scandinavian Actuarial Journal, vol. 2013, no. 2, pp. 140-162, 2013.

[22] L. He and Z. Liang, "Optimal dynamic asset allocation strategy for ELA scheme of DC pension plan during the distribution phase," Insurance: Mathematics and Economics, vol. 52, no. 2, pp. 404-410, 2013.

[23] T. R. Bielecki and S. R. Pliska, "Risk sensitive asset management with transaction costs," Finance and Stochastics, vol. 4, no. 1, pp. $1-33,2000$.

[24] M. Dai and F. Yi, "Finite-horizon optimal investment with transaction costs: a parabolic double obstacle problem," Journal of Differential Equations, vol. 246, no. 4, pp. 1445-1469, 2009.

[25] V. R. Young, "Optimal investment strategy to minimize the probability of lifetime ruin," North American Actuarial Journal, vol. 8, no. 4, pp. 106-126, 2004.

[26] J. Grandell, Aspects of Risk Theory, Springer, Berlin, Germany, 1991.

[27] P. Azcue and N. Muler, "Optimal reinsurance and dividend distribution policies in the cramér-lundberg model," Mathematical Finance, vol. 15, no. 2, pp. 261-308, 2005.

[28] S. Watanabe and N. Ikeda, Stochastic Differential Equations and Diffusion Processes, Elsevier, 1981. 
[29] M. G. Crandall, H. Ishii, and P.-L. Lions, "User's guide to viscosity solutions of second order partial differential equations," Bulletin of the American Mathematical Society, vol. 27, no. 1, pp. 1-67, 1992.

[30] H. Pham, Continuous-time Stochastic Control and Optimization with Financial Applications, vol. 1, Springer, 2009.

[31] F. E. Benth, K. H. Karlsen, and K. Reikvam, "Portfolio optimization in a Lévy market with intertemporal substitution and transaction costs," Stochastics, vol. 74, no. 3-4, pp. 517-569, 2002.

[32] G. Barles and C. Imbert, "Second-order elliptic integrodifferential equations: viscosity solutions' theory revisited," Annales de l'Institut Henri Poincare. Annales: Analyse Non Lineaire/Nonlinear Analysis, vol. 25, no. 3, pp. 567-585, 2008.

[33] M. J. P. Magill and G. M. Constantinides, "Portfolio selection with transactions costs," Journal of Economic Theory, vol. 13, no. 2, pp. 245-263, 1976.

[34] F. Dufresne and H. U. Gerber, "Risk theory for the compound poisson process that is perturbed by diffusion," Insurance Mathematics and Economics, vol. 10, no. 1, pp. 51-59, 1991.

[35] H. Yang and L. Zhang, "Optimal investment for insurer with jump-diffusion risk process," Insurance: Mathematics and Economics, vol. 37, no. 3, pp. 615-634, 2005.

[36] P. E. Protter, Stochastic Integration and Differential Equations: Version 2.1, vol. 21, Springer, 2004.

[37] S. Browne, "Optimal investment policies for a firm with a random risk process: exponential utility and minimizing the probability of ruin," Mathematics of Operations Research, vol. 20, no. 4, pp. 937-958, 1995.

[38] H. M. Soner, "Optimal control of jump-markov processes and viscosity solutions," in Stochastic Differential Systems, Stochastic Control Theory and Applications, pp. 501-511, Springer, 1988. 


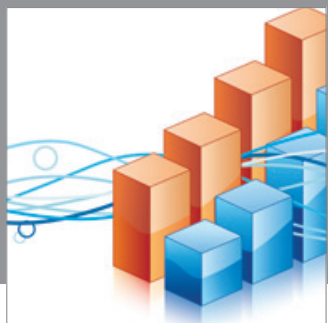

Advances in

Operations Research

mansans

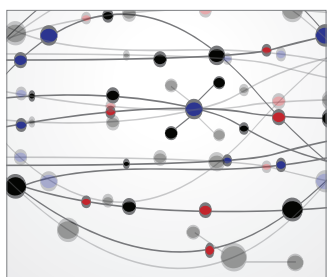

The Scientific World Journal
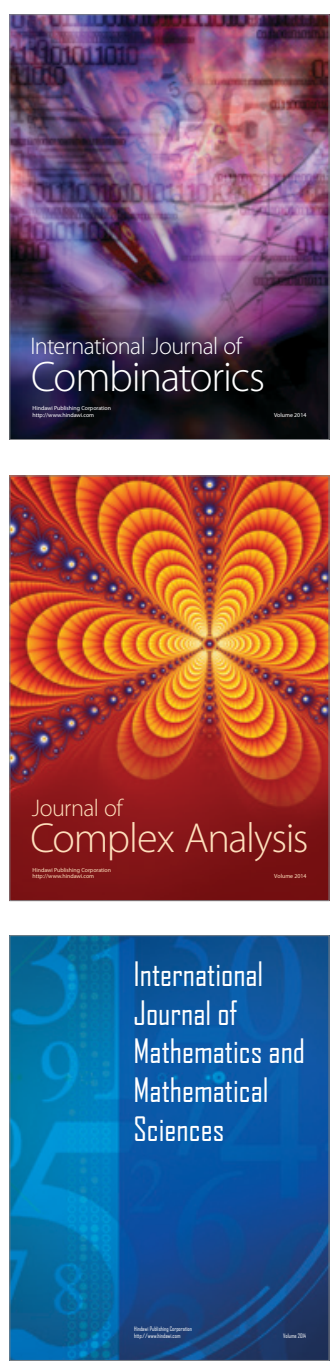
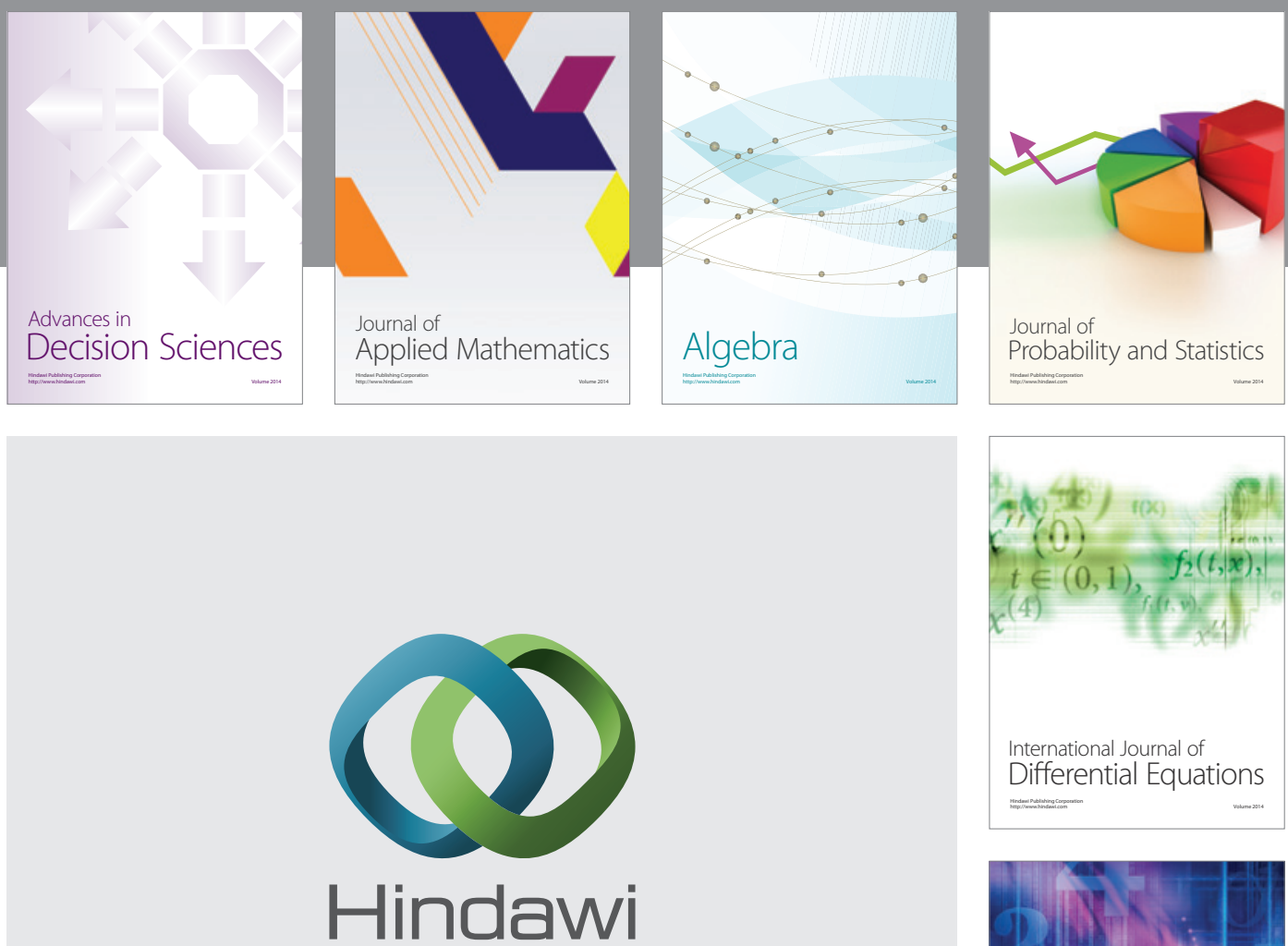

Submit your manuscripts at http://www.hindawi.com
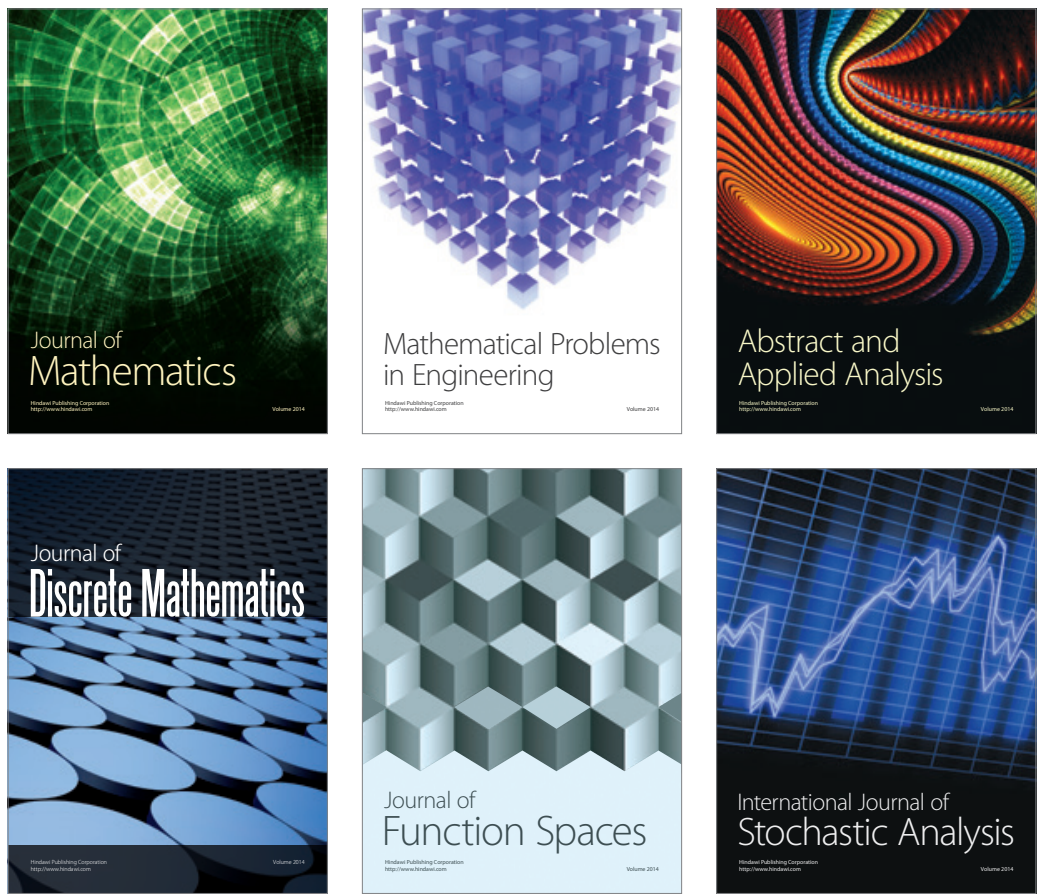

Journal of

Function Spaces

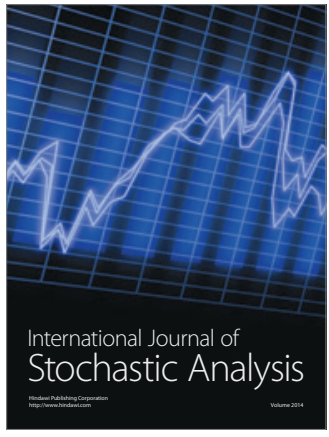

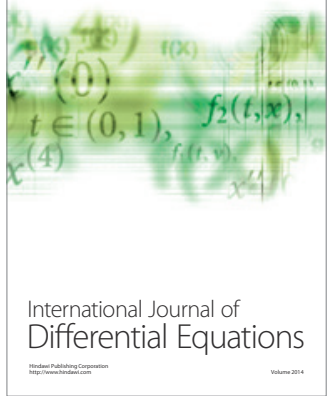
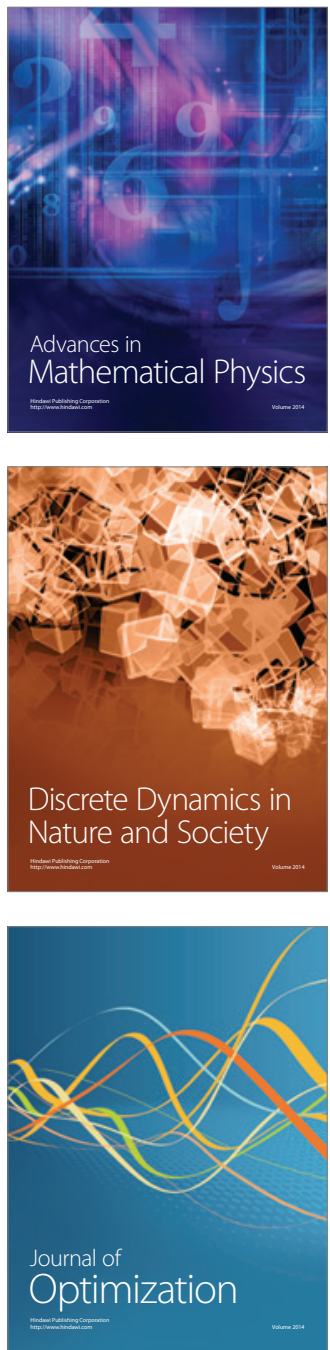